\title{
Dynamic Data Driven Applications Systems (DDDAS) - A Transformative Paradigm
}

\author{
Frederica Darema \\ National Science Foundation, 4201 Wilson Boulevard, Arlington, VA, 22230, USA \\ fdarema@nsf.gov
}

\begin{abstract}
The Dynamic Data Driven Applications Systems (DDDAS), paradigm entails the ability to dynamically incorporate data into an executing application simulation, and in reverse, the ability of applications to dynamically steer measurement processes. The ability to augment the traditional application modeling with such dynamic data inputs, acquired in real time, online, or existing as archival data, creates the possibility of entirely new capabilities through improved applications modeling, improved instrumentation methods, and systems management methods, enhancing the analysis and prediction capabilities of application simulations, improving the efficiency of simulations and the effectiveness of measurement systems, including providing powerful methodology of management of heterogeneous sensor networks. The dynamic integration of the computational and measurement aspects in DDDAS environments entail a unified of the computational and instrumentation application platform. In this presentation we will discuss the ensuing DDDAS computational model in the context of examples of novel capabilities enabled through its implementation in many application areas.

An overview of the state of the art will be presented. A discussion of how to connect U.S. and European or Asian DDDAS-like projects will be initiated for all present at the DDDAS workshop.
\end{abstract}

